\title{
Discovery of X-ray pulsations in the Be/X-ray binary IGR J06074+2205
}

\author{
P. Reig ${ }^{1,2}$ and A. Zezas ${ }^{2,3}$ \\ ${ }^{1}$ IESL, Foundation for Research and Technology-Hellas, 71110 Heraklion, Greece \\ 2 Physics Department, University of Crete, 71003 Heraklion, Greece \\ e-mail: pau@physics.uoc.gr \\ ${ }^{3}$ Harvard-Smithsonian Center for Astrophysics, 60 Garden Street, Cambridge, MA 02138, USA
}

Received 22 December 2017 / Accepted 12 February 2018

\begin{abstract}
Context. IGR J06074+2205 is a poorly studied X-ray source with a Be star companion. It has been proposed to belong to the group of $\mathrm{Be} / \mathrm{X}$-ray binaries (BeXBs). In BeXBs, accretion onto the neutron star occurs via the transfer of material from the Be star's circumstellar disk. Thus, in the absence of the disk, no X-ray should be detected.

Aims. The main goal of this work is to study the quiescent X-ray emission of IGR J06074+2205 during a disk-loss episode.

Methods. We obtained light curves at different energy bands and a spectrum covering the energy range $0.4-12 \mathrm{keV}$. We used Fourier analysis to study the aperiodic variability and epoch folding methods to study the periodic variability. Model fitting to the energy spectrum allowed us to identify the possible physical processes that generated the X-rays.

Results. We show that at the time of the XMM-Newton observation, the decretion disk around the Be star had vanished. Still, accretion appears as the source of energy that powers the high-energy radiation in IGR J06074+2205. We report the discovery of X-ray pulsations with a pulse period of $373.2 \mathrm{~s}$ and a pulse fraction of $\sim 50 \%$. The $0.4-12 \mathrm{keV}$ spectrum is well described by an absorbed power law and blackbody components with the best fitting parameters: $N_{\mathrm{H}}=(6.2 \pm 0.5) \times 10^{21} \mathrm{~cm}^{-2}, k T_{\mathrm{bb}}=1.16 \pm 0.03 \mathrm{keV}$, and $\Gamma=1.5 \pm 0.1$. The absorbed X-ray luminosity is $L_{\mathrm{X}}=1.4 \times 10^{34} \mathrm{erg} \mathrm{s}^{-1}$ assuming a distance of $4.5 \mathrm{kpc}$.

Conclusions. The detection of X-ray pulsations confirms the nature of IGR J06074+2205 as a BeXB. We discuss various scenarios to explain the quiescent X-ray emission of this pulsar. We rule out cooling of the neutron star surface and magnetospheric emission and conclude that accretion is the most likely scenario. The origin of the accreted material remains an open question.
\end{abstract}

Key words. stars: emission-line, $\mathrm{Be}-\mathrm{X}$-rays: binaries - stars: neutron

\section{Introduction}

IGRJ06074+2205 was discovered by INTEGRAL/JEM-X during public observations of the Crab region that took place on 15 and 16 February 2003 (Chenevez et al. 2004). The source was detected with a flux of $\sim 22 \mathrm{mCrab}( \pm 2 \mathrm{mCrab})$ in the energy range $3-20 \mathrm{keV}$. To localize the $\mathrm{X}$-ray source and determine the correct counterpart, Tomsick et al. (2006) obtained a $5 \mathrm{ks}$ observation with the Chandra X-ray Observatory on 2 December 2006. The Chandra flux was $2 \times 10^{-12} \mathrm{erg} \mathrm{cm}^{-2} \mathrm{~s}^{-1}$ in the energy range $0.3-10 \mathrm{keV}$. The energy spectrum could be fitted with an absorbed power-law with $N_{H}=(6 \pm 2) \times 10^{22} \mathrm{~cm}^{-2}$ and a photon index of $1.3 \pm 0.8$ ( $90 \%$ confidence errors). The absorbed flux was nearly 60 times lower than the value at the time of the INTEGRAL observation. The Chandra observation also allowed the confirmation of the Be star suggested by Halpern \& Tyagi (2005) as the correct optical counterpart.

Optical spectroscopic observations performed between 2006 and 2010 revealed a fast-rotating $(v \sin i \approx 260)$ B0.5Ve star located at $\sim 4.5 \mathrm{kpc}$ (Reig et al. 2010). The star displayed variability in the $\mathrm{H} \alpha$ line on timescales of months. Changes were seen both in the line profile and the line intensity. Even though $\mathrm{X}$-ray pulsations were not detected, the available data suggested that IGR J06074+2205 is a Be/X-ray binary (BeXB). BeXBs constitute the most numerous subgroup of high-mass $\mathrm{X}$-ray binaries and consist of a $\mathrm{Be}$ star and a neutron star
(Paul \& Naik 2011; Reig 2011). The mass donor in these systems is a relatively massive $\left(\gtrsim 10 M_{\odot}\right)$ and fast-rotating $(\gtrsim 80 \%$ of break-up velocity) star, whose equator is surrounded by a disk formed from photospheric plasma ejected by the star. $\mathrm{H} \alpha$ in emission is typically the dominant feature in the spectra of Be stars and the reason for the letter " $\mathrm{e}$ " in the spectral type identification. In addition to emission lines, Be stars also show variable polarized light and infrared excess, i.e., more near-IR emission than a normal B-type star of the same spectral type. The disk forms and dissipates on timescales of years. Because the physical conditions in the disk change substantially during the formation and dissipation phases, so do the $\mathrm{H} \alpha$ intensity and the optical and infrared photometric colors. Thus the disk is responsible for the long-term optical and infrared variability of the system. Also, since the main source of matter available for accretion is the circumstellar disk, also known as the decretion disk, the X-ray variability is expected to be closely linked to the state of the disk. In particular, when the disk disappears completely, the mass transfer toward the neutron star should halt and the X-ray emission should cease. However, at least three systems have shown X-ray emission during disk-loss phases: 1A 0535+262 (Negueruela et al. 2000), IGR J21343+4738 (Reig \& Zezas 2014), and SAX J2103.5+4545 (Reig et al. 2014). All three show X-ray pulsations during these observations. In addition, many more sources have been detected in a quiescent state at luminosities $L_{\mathrm{X}} \lesssim 10^{34} \mathrm{erg} \mathrm{s}^{-1}$ (Tsygankov et al. 2017b). The 
origin of the X-ray emission and of the accreted material in this state remain unknown.

In this work, we present an X-ray analysis during one of these disk-loss episodes giving us the rare opportunity to study a $\mathrm{BeXB}$ in the quiescent phase where the reservoir of accretion gas is exhausted. We report the discovery of X-ray pulsations with a spin period of $373.2 \mathrm{~s}$.

\section{Observations}

\subsection{X-ray band}

IGR J06074+2205 was observed by XMM-Newton on 29 September 2017 during revolution 3261. The observation (ObsID $0794581301)$ started at 11:49 h UT and lasted for $\sim 40 \mathrm{ks}$. The XMM-Newton Observatory (Jansen et al. 2001) includes three $1500 \mathrm{~cm}^{2}$ X-ray telescopes each with a European Photon Imaging Camera (EPIC) at the focus. Two of the EPIC imaging spectrometers use metal oxide semi-conductor (MOS) CCDs (Turner et al. 2001) and one uses pn-CCDs (Strüder et al. 2001). Reflection Grating Spectrometers (den Herder et al. 2001) are located behind two of the telescopes while the $30-\mathrm{cm}$ optical monitor (OM) instrument has its own optical/UV telescope (Mason et al. 2001). Data were reduced using the XMM-Newton Science Analysis System (SAS version 16.1).

Prior to the extraction of the data products, we processed the observation data files (ODFs) to obtain calibrated and concatenated event lists. We filtered the event-list file to extract EPIC-pn events with a pixel pattern in the range 0-4 (single and doubles) and EPIC-MOS events with patterns 0-12. For the spectral analysis, we used the more strict selection criterion $F L A G=0$, which omits parts of the detector area like border pixels and columns with higher offset. The first $16240 \mathrm{~s}$ are affected by flaring particle background and were discarded for the spectral analysis. For the timing analysis, we performed a barycentric correction, that is, the arrival time of the photons was shifted to the barycentre of the solar system. To generate the light curves and spectrum, we extracted events from a circular region with radius 40 arcsec. This size is the maximum allowed to avoid including the CCD edge. It includes more than $90 \%$ of the encircled energy of $1.5 \mathrm{keV}$ photons. To select the background region, we chose a region of the same size $\sim 2$ arcmin away from the source.

The EPIC instruments were used in the full frame mode. In this mode, all pixels of all CCDs are read out and thus the full field of view is covered. The highest possible time resolution in this mode is $73.4 \mathrm{~ms}$ for EPIC-pn and $2.6 \mathrm{~s}$ for MOS. IGR J06074+2205 is the brightest source detected in the field of view. The source region is free of pile-up as demonstrated by the fact that the observed distribution of counts as a function of the PI channel of single and double events agrees with the expected one. Using the SAS task epatplot we find that the $0.5-2.0 \mathrm{keV}$ observed-to-model singles and doubles pattern fractions ratios are consistent with 1.0 within statistical errors $(0.992 \pm 0.027$ and $1.011 \pm 0.038$ for single and double events, respectively).

We mainly used data from the EPIC-pn camera because it is the instrument with the highest effective area and the highest time resolution. Nevertheless, we checked that we obtained consistent results with different cameras.

\subsection{Optical band}

We observed the source with the OM/XMM-Newton in the fast mode. We extracted light curves with a total duration of
2000 s for bands $U, B$, and $V$ and 8800 s for UV bands $U V W 1$ $\left(\lambda_{\mathrm{eff}}=291 \mathrm{~nm}\right), U V W 2\left(\lambda_{\mathrm{eff}}=212 \mathrm{~nm}\right)$, and $U V M 2\left(\lambda_{\mathrm{eff}}=\right.$ $231 \mathrm{~nm}$ ). Timing analyses on each of these light curves did not reveal any variability. The average magnitudes are: $U=12.43$, $B=13.04$, and $V=12.34$.

The spectroscopic observations were made with the $1.3 \mathrm{~m}$ telescope of the Skinakas Observatory (Crete, Greece). We used $2048 \times 204813.5 \mu \mathrm{m}$ pixel ANDOR IKON and a $13021 \mathrm{~mm}^{-1}$ grating, giving a nominal dispersion of $\sim 0.94 \AA \mathrm{pixel}^{-1}$. Spectra of comparison lamps were taken before each exposure in order to account for small variations of the wavelength calibration during the night. To ensure an homogeneous processing of the spectra, they were normalized with respect to the local continuum, which was rectified to unity by employing a spline fit. A disk-loss episode is identified when the $\mathrm{H} \alpha$ line reverts from emission into absorption. In the absence of the disk, we expect to observe the typical photospheric absorption line of an earlytype star. We report here spectroscopic observations taken close in time to the XMM-Newton observation. For a long-term optical variability study of the source see Reig et al. (2016).

\section{Results}

The optical/IR emission lines in Be stars arise from radiative processes in the disk. In particular, the $\mathrm{H} \alpha$ line is optically thick and formed by recombination. It is the strongest feature and forms over a large region of the disk at its outermost parts. Consequently, $\mathrm{H} \alpha$ emission should disappear when the disk dissipates. The most common way to quantify the strength of the line is the equivalent width. Negative values indicate emission profiles, while positive values correspond to absorption dominated profiles, i.e., with the absence of the disk. Positive values of the $\mathrm{EW}(\mathrm{H} \alpha)$ between 0 and the expected value of a purely photospheric line, for example, $\approx+3.5 \AA$ for B0.5V Be star (Jaschek \& Jaschek 1987), indicate that the line is partially filled with emission.

Figure 1 displays three $\mathrm{H} \alpha$ line profiles of IGR J06074+2205 at different epochs. The top and middle panels show the $\mathrm{H} \alpha$ line 17 days before (12 September 2017) and 15 days after (13 October 2017) the XMM-Newton observation. Both observations show $\mathrm{H} \alpha$ in absorption at a level of $\mathrm{EW}(\mathrm{H} \alpha)=+2.3 \AA$, indicating that the disk had vanished at the time of the X-ray observation, although some weak residual emission cannot be completely ruled out. In fact, the most recent observation, in November 2017 showed $\operatorname{EW}(\mathrm{H} \alpha)=-0.7 \AA$ (bottom panel in Fig. 1), suggesting that the disk has begun to form again.

\subsection{Discovery of $X$-ray pulsations}

Although the material that feeds the neutron star was exhausted, X-ray emission from IGR J06074+2205 is clearly detected. To investigate the X-ray variability, we extracted a $1 \mathrm{~s}$ binned light curve in the energy range $0.4-12 \mathrm{keV}$.

The average $0.4-12 \mathrm{keV}$ source count rate after background subtraction was $0.970 \pm 0.006$ counts $^{-1}$. The average background rate for the entire $38.8 \mathrm{ks}$ observation was $0.036 \pm$ 0.001 counts $\mathrm{s}^{-1}$, but it was larger at the beginning of the observation owing to enhanced flaring particle background. The average background count rate during the first $16 \mathrm{ks}$ was $0.062 \pm$ 0.002 counts $\mathrm{s}^{-1}$, while for the rest of the observation it remained at $0.017 \pm 0.001$ counts $^{-1}$. The X-ray light curve exhibited moderate variability on time scales of a few kiloseconds. The root-mean-square measured in the $1 \mathrm{~s}$ binned light curve was $43 \pm 1 \%$. 


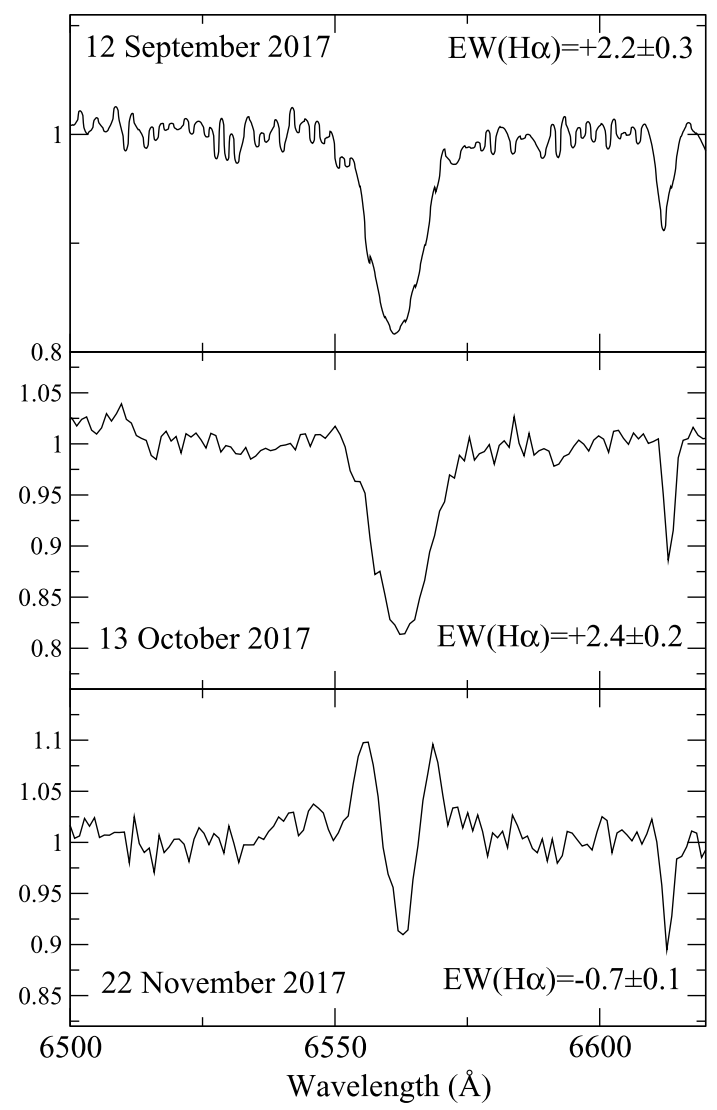

Fig. 1. $\mathrm{H} \alpha$ line profile before and after the X-ray observations on 29 September 2017.

To search for pulsations, we run a fast Fourier transform (FFT) and produce a Leahy-normalized (Leahy et al. 1983) power spectrum covering the frequency interval $3.05 \times 10^{-5}$ $0.5 \mathrm{~Hz}$ in 32768 bins (Fig. 2). Three peaks are clearly seen in the power spectrum at $v_{1}=0.002679 \mathrm{~Hz}, v_{2}=0.005356 \mathrm{~Hz}$, and $v_{3}=0.008039 \mathrm{~Hz}$. All peaks are significant above $5 \sigma$ level, indicating that there is a distinct periodic signal in the data. Because there is a strong red noise component (see Sect. 3.2), the power spectrum was normalized to unity and the result multiplied by 2 . In this way we ensure that the power spectral continuum agrees with the expected white noise power in the Leahy normalization (Leahy et al. 1983; Leahy 1987). This is a necessary step prior to the calculation of the significant level.

The power of the lowest frequency peak is distributed over several bins, making this peak appear less significant than the second peak. However, the ratios of the peak frequencies imply that the second peak cannot represent the fundamental frequency. If $v_{2}$ was the fundamental frequency then $v_{3}$ would be the first harmonic, hence $v_{3} / v_{2}=2$. Clearly, $v_{3} / v_{2} \neq 2$. In contrast, if $v_{1}$ is the main periodicity, then $v_{2} / v_{1}=2$ and $v_{3} / v_{1}=3$ as indeed it is the case. Therefore $v_{2}$ and $v_{3}$ are the first and second harmonics of the main signal $v_{1}$ at $0.002685 \mathrm{~Hz}$. This frequency corresponds to a period of $\sim 372.4 \mathrm{~s}$.

We also searched for periodic signal using an epoch-folding technique where the data is folded over a period range (Leahy 1987; Larsson 1996). For each trial period, the $\chi^{2}$ statistic is calculated. If the data contain a periodic signal, then a peak stands out in the $\chi^{2}-P_{\text {trial }}$ plot. We used the task efsearch of the XRONOS package and found a best-fit period at 373.2 s (Fig 2, lower panel).

To improve the estimation of the pulse period, we next applied a phase fitting technique. We divided the light curve
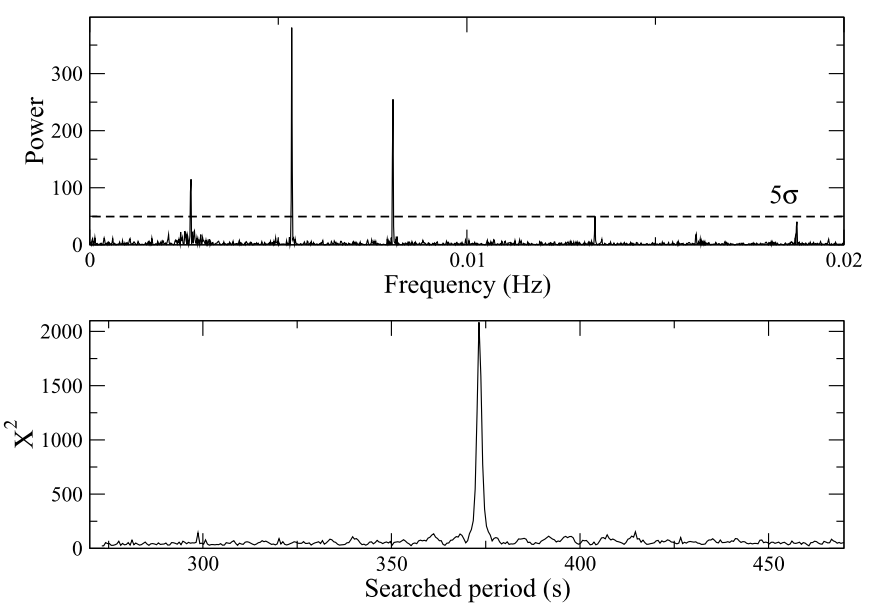

Fig. 2. Upper panel: EPIC-pn power spectrum and $\sim 5 \sigma$ significance level. Lower panel: $\chi^{2}$ maximization after folding the data over a range of periods (epoch folding).

into 8 segments, each with a length equal to 12 pulse periods approximately and calculated a folded pulse profile for each segment with a common epoch and period. These profiles were cross-correlated with a template obtained by folding the entire light curve onto the trial period. The resulting phase delays were fitted with a linear function, whose slope provides the correction needed to be applied to the trial period. We then adjusted the period and repeated the procedure until the phase delay exhibited no net trend with time throughout the observation. The best-fit period was $373.226 \pm 0.013 \mathrm{~s}$, where the error was estimated from the uncertainty on the first-order term of a linear fit to the phase delays.

Next, we extracted light curves at various energy bands and folded the best-fit pulse period onto these light curves to obtain the pulse profiles (Fig. 3). The pulse profile is complex with a multi-peak structure. The pulse fraction, defined as $P F=\left(I_{\max }-I_{\min }\right) /\left(I_{\max }+I_{\min }\right)$, where $I_{\min }$ and $I_{\max }$ are background-corrected count rates at the pulse profile minimum and maximum, does not strongly depend on energy and varies in the range $50-60 \%$.

\subsection{Power spectrum}

The ability to detect a narrow feature in the power spectrum, i.e., pulsations, is highly reduced when any kind of averaging (adjacent bins or segments) is carried out (van der Klis 1989). This is the reason that we used full time resolution and no segment division in Sect. 3.1 and Fig. 2. However, a study of the peaked and broad-band noise may also be of interest. In this section, we examine the structure of the power spectrum to identify possible noise components.

To obtain the power spectrum shown in Fig. 4, we proceeded as follows: we extracted $\Delta t \mathrm{~s}$ binned light curves in the energy range $0.4-12 \mathrm{keV}$. The light curve was divided into segments of duration $T$ and a Fast Fourier Transform was computed for each segment. The final power spectrum is the average of all the power spectra obtained for each segment. The power spectra were logarithmically rebinned in frequency and normalized such that the integral between two frequencies gives the squared rms fractional variability. The expected white noise level was subtracted. We tried different combinations of $\Delta t$ and $T$ and found that above $v \sim 0.1 \mathrm{~Hz}$ the power spectrum is white-noise dominated. Therefore, we used $\Delta t=1 \mathrm{~s}$ and $T=8192 \mathrm{~s}$ covering a frequency range $0.00012-0.5 \mathrm{~Hz}$. 


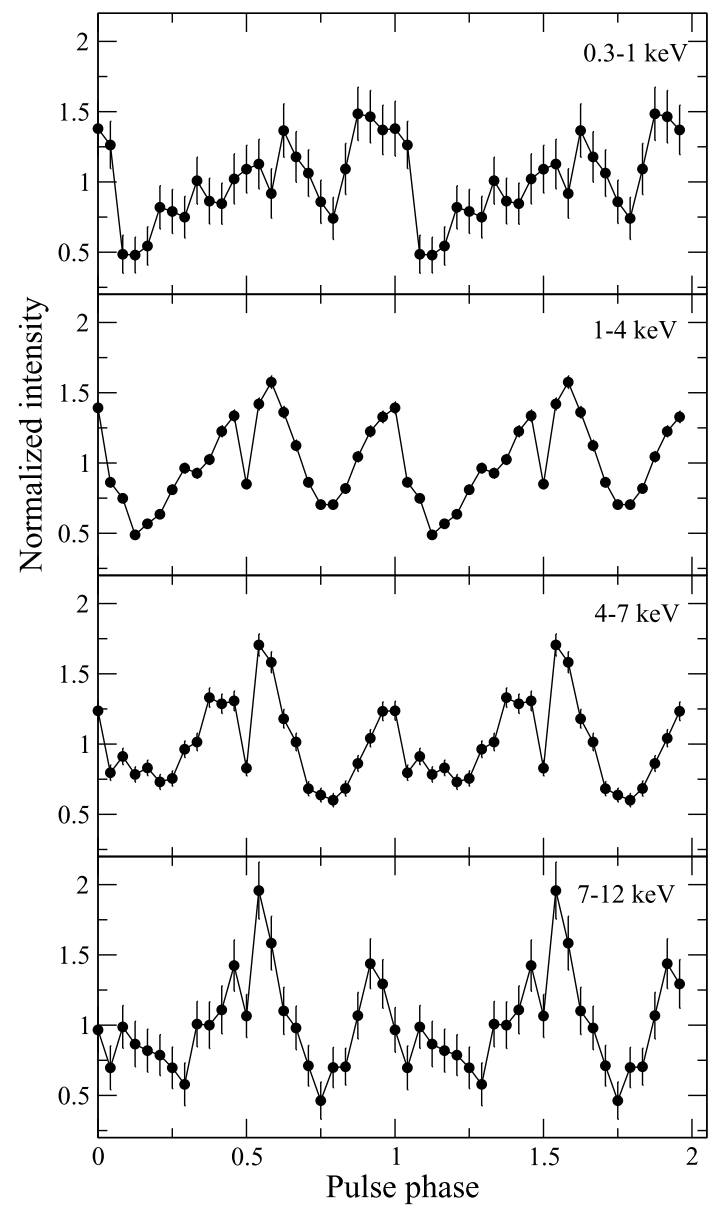

Fig. 3. Normalized pulse profiles at different energies.

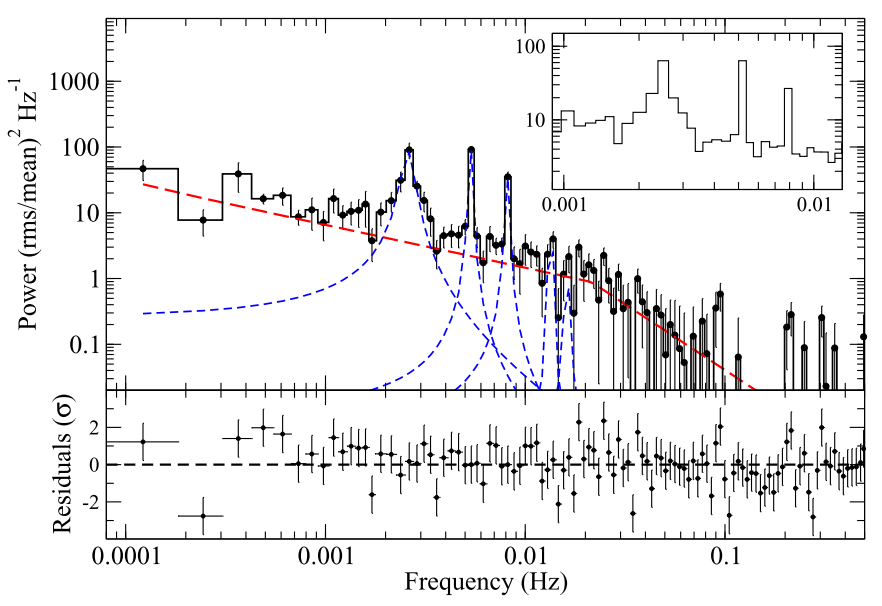

Fig. 4. Power spectrum of IGR J06074+2205 (circles) and model components, which consist of a broken power law (red dashed line) and multi Lorentzian profiles (blue lines). Bottom panel: residuals in number of sigmas with error bars of size one. The inset shows with detail the broadening at the base of the periodic signal.

The power spectrum shows strong red noise. The spectral continuum can be described by two zero-centred Lorentzians with widths $0.0019 \pm 0.0006 \mathrm{~Hz}$ and $0.023 \pm 0.006 \mathrm{~Hz}$, respectively (reduced $\chi^{2}=1.28$ for 98 degrees of freedom) or with a broken power law with indices $\Gamma_{1}=0.65 \pm 0.05$ and $\Gamma_{2}$ fixed to 2 , and a break at $v_{\text {break }}=0.024 \pm 0.004 \mathrm{~Hz}$ (reduced $\chi^{2}=1.26$ for 98 degrees of freedom). The spikes caused by the pulse period and its harmonics were fitted with Lorentzian profiles fixed at the expected frequencies. The rms in the frequency range $0.0001-0.1 \mathrm{~Hz}$ is $40 \%$.

An interesting feature of the power spectrum is the strong coupling between the periodic and aperiodic variability (see Sect. 4.1), which manifests itself as a broadening at the base of the harmonics (Lazzati \& Stella 1997; Burderi et al. 1997) and might be the reason that the power of the fundamental signal is smaller than the harmonics (Fig. 2).

\subsection{Spectral analysis}

We extracted an EPIC-pn energy spectrum using the same source and background regions as for the timing analysis and filtering criteria described. For the spectral analysis we further cleaned the data by accepting only the good times when sky background was low. We rebinned the energy spectra by requiring at least 25 counts for each energy bin.

Single-component models do not give good fits. An absorbed blackbody model leaves residuals with a sinusoidal structure and differences between the data and the model of $\gtrsim 2 \sigma$ in $17 \%$ of the bins. This model gives a reduced $\chi^{2}=2$ for 150 degrees of freedom (dof). A single power law does not describe the spectrum either with $\chi_{\text {red }}^{2}=4.5$ for 150 dof. The addition of a cutoff provides an acceptable fit $\left(\chi_{\text {red }}^{2}=1.07\right.$ for 149 dof). The best-fit spectral parameters for the power-law plus cutoff model are: $N_{\mathrm{H}}=(4.7 \pm 0.3) \times 10^{21} \mathrm{~cm}^{-2}, \Gamma=-0.40 \pm 0.09$, and $E_{\text {cut }}=2.1 \pm 0.1 \mathrm{keV}$. These values are significantly different from the typical values in accreting pulsars and are hard to interpret. The photon index and cutoff energy in accreting pulsars vary typically in the ranges $0.5-1.5 \mathrm{keV}$ and $10-20 \mathrm{keV}$, respectively.

The combination of a blackbody and a power law provides an excellent fit with $\chi_{\text {red }}^{2}=1.01$ for 148 dof. In this case, the following best-fit parameters are found: $N_{\mathrm{H}}=(6.2 \pm 0.5) \times 10^{21} \mathrm{~cm}^{-2}$, $k T_{\mathrm{bb}}=1.16 \pm 0.03 \mathrm{keV}$, and $\Gamma=1.5 \pm 0.1$. The uncertainties correspond to $1 \sigma$ errors. The normalization of the blackbody component agrees with an emitting region of $R_{\mathrm{bb}}=200 \pm 50 \mathrm{~m}$ in size. The column density was obtained assuming abundances given in Anders \& Grevesse (1989) and cross-sections from Verner et al. (1996). Figure 5 shows the EPIC-pn spectrum and the two-component model described above. The total absorbed X-ray luminosity in the energy range $0.2-12 \mathrm{keV}$ is $1.5 \times 10^{34} \mathrm{erg} \mathrm{s}^{-1}\left(1.8 \times 10^{34} \mathrm{erg} \mathrm{s}^{-1}\right.$, unabsorbed $)$, assuming a distance of $4.5 \mathrm{kpc}$ (Reig et al. 2010). The contribution from each model component to the total luminosity is $L_{\mathrm{bb}}=$ $9.3 \times 10^{33} \mathrm{erg} \mathrm{s}^{-1}$ and $L_{\mathrm{pl}}=8.6 \times 10^{33} \mathrm{erg} \mathrm{s}^{-1}$. The contribution of the blackbody component to the source luminosity amounts to $\sim 52 \%$. This value is somewhat larger but comparable to that measured in other BeXBs (La Palombara \& Mereghetti 2006, 2007; La Palombara et al. 2009). We found no evidence for an iron emission line. The upper limit on the equivalent width of a narrow $(\sigma=0.1 \mathrm{keV}) 6.4 \mathrm{keV}$ emission line is $30 \mathrm{eV}$.

\section{Discussion}

We have carried out an X-ray timing and spectral analysis of IGR J06074+2205 at a time when no X-ray emission should be expected. The circumstellar (decretion) disk around the $\mathrm{Be}$ star's equator had presumably vanished at the time of the XMMNetwon observation. Not only did we detect the source at a relatively high level $\left(10^{34} \mathrm{erg} \mathrm{s}^{-1}\right)$, but we also report X-ray pulsations for the first time in this source. 


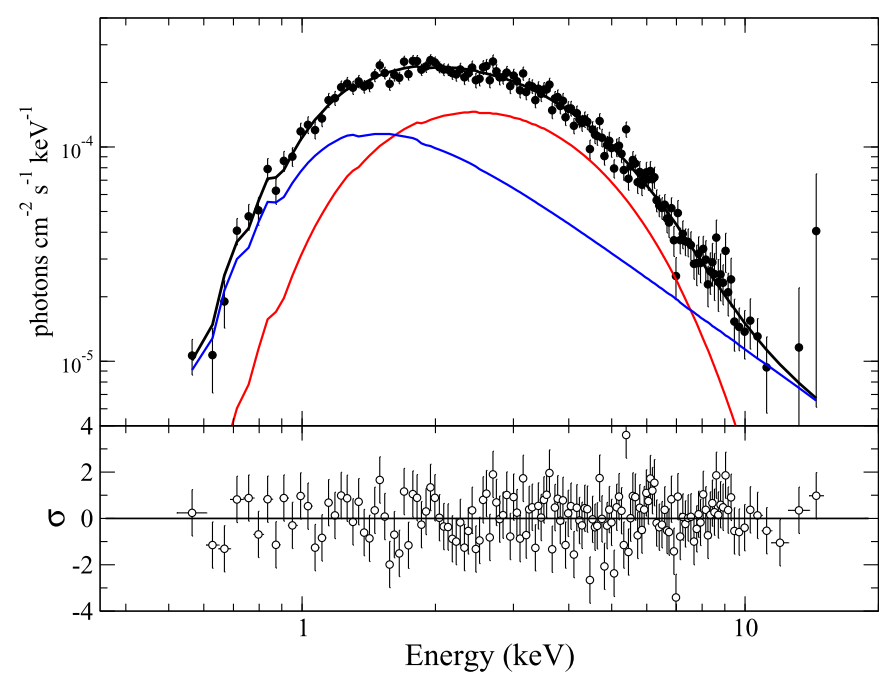

Fig. 5. EPIC-pn spectrum of IGR J06074+2205 (circles) and best-fit model (black line), which consists of a blackbody (red line) and a power law (blue line). Bottom panel: residuals in terms of sigmas with error bars of size one.

\subsection{Coupling between the periodic and aperiodic variability}

The power spectrum of IGR J06074+2205 is characterized by the narrow peaks of the periodic modulation and its harmonics and by a noise component whose power increases toward lower frequencies. Red noise is a common characteristic in all kind of accreting binaries. It is attributed to instabilities in the accretion flow as it approaches the compact object. If these instabilities are produced after the flow is trapped by the magnetic field, they should be affected by the periodic modulation (Lazzati \& Stella 1997; Burderi et al. 1997). The power spectrum of IGR J06074+2205 is very similar to that of SMC X-1 (Burderi et al. 1997), Vela X-1 and 4U 1145-62 (Lazzati \& Stella 1997) and shows a strong coupling of the red noise with the periodic modulation. This coupling manifests observationally by the broadening of the wings of the narrow peaks due to the periodic modulation and becomes more apparent when the red-noise power increases short-ward of the pulsar frequency, as is the case in IGR J06074+2205.

\subsection{Origin of the quiescent $X$-ray emission}

In this section, we discuss the possible mechanisms that may account for the X-ray emission of accreting pulsars at low luminosities. Accretion can explain the X-ray luminosity observed in X-ray pulsars in a large range from $10^{34}$ to $10^{38} \mathrm{erg} \mathrm{s}^{-1}$. Two are the basic ingredients in the accretion mechanism: a source of matter and a strong gravitational potential. In accreting pulsars, the magnetic field also plays a fundamental role because together with the mass accretion rate it defines the size of the magnetosphere. In BeXBs, the source of matter is the circumstellar disk around the Be star, while the source of strong gravity is the neutron star.

Accretion may cease if the mass accretion rate $\dot{M}$ goes below a critical value. When the radius of the magnetosphere, $r_{\mathrm{m}}$ grows beyond the co-rotation radius, $r_{\mathrm{co}}$ (at which the angular velocity of Keplerian motion is equal to that of the neutron star), the centrifugal force prevents material from entering the magnetosphere. This is known as the propeller effect (Illarionov \& Sunyaev 1975) or centrifugal inhibition of accretion (Stella et al. 1986). While $r_{\mathrm{co}}$ does not depend on quantities that vary substantially with the mass accretion rate, $r_{\mathrm{co}}=\left(G M_{\mathrm{NS}} P_{\mathrm{spin}}^{2} / 4 \pi\right)^{1 / 3}$, the radius of the magnetosphere strongly depends on the mass accretion rate $r_{\mathrm{m}}=\left(\mu^{4} /\left(2 G M_{\mathrm{NS}} \dot{M}^{2}\right)\right)^{1 / 7}$, where $\mu$ is the magnetic moment. If $\dot{M}$ is highly reduced, then the inequality may reverse and become $r_{\mathrm{m}}>r_{\mathrm{co}}$, in which case the propeller mechanism sets in. Thus the propeller effect is expected to occur at the end of an X-ray outburst or when there is no supply of matter.

The minimum X-ray luminosity below which the propeller effect sets in can be determined by equating the co-rotation radius to the radius of the magnetosphere (Stella et al. 1986; Campana et al. 2002)

$L_{\mathrm{min}}\left(R_{\mathrm{NS}}\right)=3.9 \times 10^{37} k^{7 / 2} B_{12}^{2} P_{\text {spin }}^{-7 / 3} M_{1.4}^{-2 / 3} R_{6}^{5} \mathrm{erg} \mathrm{s}^{-1}$

where $M_{1.4}$ and $R_{6}$ are the mass and radius of the neutron star in units of $1.4 M_{\odot}$ and $10^{6} \mathrm{~cm}$, respectively, $P_{\text {spin }}$ is the spin period in seconds, $B_{12}$ is the magnetic field in units of $10^{12} \mathrm{G}$, and $k$ is the ratio of the magnetosphere to the Alfen radius (typically $k=$ 0.5 for disk accretion). For typical values of the magnetic field (a few times $\left.10^{12} \mathrm{G}\right), L_{\text {min }}$ is of the order of $\sim 10^{36} \mathrm{erg} \mathrm{s}^{-1}$ in rapid rotating pulsars $\left(P_{\text {spin }} \sim 1 \mathrm{~s}\right)$. For long pulsing systems $\left(P_{\text {spin }} \sim\right.$ $100 \mathrm{~s})$, it is significantly lower, $L_{\min } \sim 10^{32}-10^{33} \mathrm{erg} \mathrm{s}^{-1}$.

When the propeller effect is at work, X-rays may be generated through magnetospheric accretion (Corbet 1996; Campana et al. 2001). In this scenario, the matter in the accretion flow is halted at the magnetospheric boundary and would not reach the neutron star surface. On first approximation, the accreted luminosity will be driven by the same mass transfer as in the accretor regime but with the magnetospheric radius replacing the neutron star radius $L_{\mathrm{m}} \approx G M_{\mathrm{X}} \dot{M} / r_{\mathrm{m}}$. The maximum luminosity in this regime will occur when the magnetospheric radius equals the corotation radius (Campana et al. 2002),

$L_{\mathrm{mag}}\left(r_{\mathrm{co}}\right)=L_{\mathrm{min}}\left(R_{\mathrm{NS}}\right) \frac{R_{\mathrm{NS}}}{r_{\mathrm{co}}}$.

Although X-ray pulsations may still be produced in this state, the pulse fraction should be lower than in the case of accretion onto the polar caps. The luminosity is expected to be very low $L_{\text {mag }} \lesssim 10^{31} \mathrm{erg} \mathrm{s}^{-1}$ for systems with $P_{\text {spin }}>100 \mathrm{~s}$.

Because BeXBs contain massive companions, one may expect that matter could still proceed from the optical star into the compact object via the stellar wind. To estimate the X-ray luminosity that results from direct accretion from a stellar wind, we assume that all the gravitational energy is converted into X-rays, $L_{\text {wind }}=G M_{\mathrm{X}} \dot{M}_{\mathrm{acc}} / R_{\mathrm{X}}$ and that the fraction of the stellar wind captured by the neutron star is (Frank et al. 2002)

$\frac{\dot{M}_{\mathrm{acc}}}{\dot{M}}=\frac{G^{2} M_{\mathrm{NS}}}{a^{2} v_{\mathrm{w}}^{4}(a)}$,

where $a$ is the orbital separation, $\dot{M}$ the mass-loss rate of the Be star, and $v_{\mathrm{w}}$ the terminal velocity of the stellar wind. Using Kepler's law to replace $a$ we finally obtain (see also Waters et al. 1988)

$L_{\text {wind }} \approx 4.7 \times 10^{37} M_{1.4}^{3} R_{6}^{-1} M_{*}^{-2 / 3} P_{\text {orb }}^{-4 / 3} \dot{M}_{-6} v_{\mathrm{w}, 8}^{-4} \mathrm{erg} \mathrm{s}^{-1}$

where $M_{*}$ is the mass of the optical star in solar masses and $P_{\text {orb }}$ is the orbital period of the binary in days. The mass-loss rate of the $\mathrm{Be}$ star is in units of $10^{-6} M_{\odot} \mathrm{yr}^{-1}$ and the wind velocity is in units of $10^{8} \mathrm{~cm} \mathrm{~s}^{-1}$. The classical supergiant X-ray binaries are believed to be powered by accretion via a strong stellar wind. 
The alternative to direct accretion from a high- or lowvelocity outflow is that accretion proceeds through magnetospheric leakage via an accretion disk (Tsygankov et al. 2017a,b, see also Syunyaev \& Shakura 1977). If the mass accretion rate is low enough that the temperature outside the magnetosphere remains below $6500 \mathrm{~K}$, i.e., the temperature at which hydrogen recombines (above $6500 \mathrm{~K}$ hydrogen is ionised), then a cold disk is formed. Accretion is expected to proceed at a low rate. The X-ray luminosity in this state is (Tsygankov et al. 2017a)

$L_{\text {disk }} \approx 7 \times 10^{33} A^{-7 / 13} k^{21 / 13} M_{1.4}^{3 / 13} R_{6}^{23 / 13} B_{12}^{12 / 13} T_{6500}^{28 / 13} \mathrm{erg} \mathrm{s}^{-1}$,

where $A$ is a parameter that depends on the location at which the viscous stress disappears as it interacts with the magnetosphere and varies in the range $0.06-1(A \approx 0.06$ if the stress disappears at $r_{\mathrm{m}}$ ). According to this scenario, if $L_{\text {min }}>L_{\text {disk }}$, then the pulsar will transit to the propeller state. Otherwise, accretion at a low rate would proceed from the cold disk.

If accretion stops completely, then the only other mechanism that can also give rise to X-rays is the cooling of the neutron star. During the accretion phase, the crust of a neutron star is heated by nuclear reactions. This heat is conducted inwards, heating the core, and outwards, where it is emitted as thermal emission from the surface. After the active accretion period, the crust of a neutron star cools by X-ray emission until it reaches thermal equilibrium with the core emission corresponding to the quiescent state (Brown et al. 1998; Rutledge et al. 2007; Wijnands \& Degenaar 2016).

In summary, there are two basic mechanisms to explain the $\mathrm{X}$-ray emission of accreting pulsars at very low luminosities, namely accretion and cooling of the neutron star surface. In either case, the physical conditions on which these mechanisms occur lead to different scenarios. Accretion may occur at the magnetosphere via a stellar wind or through an accretion disk. Likewise, the main contribution to the cooling process may come from the entire surface of the neutron star or from the polar caps. In principle, timing and spectral analysis may help distinguish between the various possibilities. In general terms, if accretion takes place, we expect a power-law dominated spectrum (due to Comptonization) and significant variability ( $\mathrm{rms}>10 \%$ ), while a thermal spectrum and white-noise-dominated power spectrum is expected in the case of cooling of the polar caps. A combination of the two cannot be ruled out.

\subsection{IGR J06074+2205}

In this section we discuss which of the possible scenarios best describes the X-ray properties of IGR J06074+2205.

First, we examine whether the system enters the propeller state, as expected by the disappearance of the source that supplies matter to the neutron star. Unfortunately, there is no direct estimate of the magnetic field of the neutron star in IGR J06074+2205, hence $L_{\min }$ (Eq. (1)) cannot be calculated. With a spin period of $373.2 \mathrm{~s}$, we would expect $L_{\min }$ to be relatively low. Assuming $B \sim 5 \times 10^{12}$, the luminosity at which the source would enter the propeller state is $L_{\min } \sim 10^{33} \mathrm{erg} \mathrm{s}^{-1}$. This is well below the observed luminosity. Reversing the argument, if the observed luminosity corresponded to the propeller state, then a magnetic field of $B \sim 2 \times 10^{13} \mathrm{G}$ would be needed, which is three times larger than the largest measured magnetic field in an accreting pulsar implied by the detection of cyclotron lines (Yamamoto et al. 2014; Walter et al. 2015). If centrifugal inhibition of accretion was at work in IGR J06074+2205, then the
$\mathrm{X}$-ray emission could in principle result from magnetospheric accretion. However, there are several reasons why this mechanism can be ruled out. When the source enters the centrifugally inhibited state, a sharp drop of one or two orders of magnitude in luminosity (from $L_{\min }$ to $L_{\mathrm{mag}}$ ) in the accreting luminosity should be observed. This drop would occur on time scales of a few days (Corbet 1996; Campana et al. 2002; Tsygankov et al. 2016; Reig \& Milonaki 2016). The X-ray luminosity expected from magnetospheric accretion is far too low even in the case of a very strong magnetic field, $L_{\mathrm{mag}} \sim 10^{30} \mathrm{erg} \mathrm{s}^{-1}$ (Eq. (2)). This low luminosity is a consequence of the strong dependence of $L_{\text {mag }}$ on $P_{\text {spin }}\left(L_{\text {mag }} \propto P_{\text {spin }}^{-3}\right)$. Second, the best-fit spectral parameters imply a small emitting radius, $R_{\mathrm{bb}} \sim 0.2 \mathrm{~km}$, which is too small to come from an extended region at $R_{\mathrm{m}} \sim 10^{9} \mathrm{~cm}$. Finally, the coupling of the periodic signal and the noise indicates that particles in the accretion flow have moved down along the magnetic field lines close to the surface of the neutron star.

The cooling of the neutron star surface also encounters a number of problems as the main mechanism producing the X-ray emission in IGR J06074+2205. The blackbody temperature and small emitting area imply that the cooling cannot occur over the entire surface of the pulsar. In fact, the large pulse fraction and broad pulse profiles indicate that the emission arises from a rotating region which is hotter (and more luminous) than the rest of the surface of the neutron star. Although nonuniform cooling can be attributed to the strong magnetic field (Geppert et al. 2006; Wijnands \& Degenaar 2016), the X-ray spectrum of IGR J06074+2205 deviates from pure thermal emission. There is excess emission above $\sim 7 \mathrm{keV}$ in the form of a power law, indicating the presence of nonthermal processes. Another result that argues against a purely thermal process in IGR J06074+2205 is the degree of variability and shape of the power spectral continuum. The XMM-Newton power spectrum shows strong low-frequency noise (red noise). White noise, that is, no dependence of power on frequency, would be expected in case of thermal cooling. Red noise is believed to be produced by aperiodic variability associated with instabilities in the accretion flow. Finally, the observed X-ray luminosity is higher than predicted from crustal heating. The X-ray luminosity expected from crustal heating depends on the time-averaged accretion rate as $L_{q} \sim 6 \times 10^{32}\left(\dot{M} / 1 \times 10^{-11}\right)$ $M_{\odot} \mathrm{yr}^{-1}$, where $\dot{M}$ is the average accretion rate including outbursts (Brown et al. 1998; Rutledge et al. 2007; Wijnands et al. 2013). Typical values of $L_{q}$ in BeXBs are well below $10^{34} \mathrm{erg} \mathrm{s}^{-1}$ (Tsygankov et al. 2017b).

Although crustal heating is not the dominating mechanism that accounts for the observed X-ray emission in IGR J06074+2205, a strong thermal component is present. The high temperature, small area, and large pulse fraction strongly suggest that the origin of this component is the polar caps. This thermal component has been observed in many, probably all, accreting X-ray pulsars (Hickox et al. 2004). In fact the values of $k T_{\mathrm{bb}}=1.16 \pm 0.03 \mathrm{keV}$ and $R_{\mathrm{bb}}=200 \pm 50 \mathrm{~m}$ agree well with those of $1 \mathrm{~A} 0536+262$ (Mukherjee \& Paul 2005), RX J0146.9+6121 (La Palombara \& Mereghetti 2006), X-Per (La Palombara \& Mereghetti 2007), and RX J1037.5-5647 (La Palombara et al. 2009). All these systems were observed to have a similar luminosity to that of IGR J06074+2205 $\left(L_{X} \lesssim 10^{35} \mathrm{erg} \mathrm{s}^{-1}\right)$ and displayed a similar spectrum, namely an absorbed power law plus blackbody emission. An important difference of IGR J06074+2205 with respect to all these systems (except for RX J1037.5-5647 for which no information could 
be found) is that at the time of the X-ray observation, they all had $\mathrm{H} \alpha$ in emission, indicating the presence of the equatorial disk (Reig et al. 2016).

In summary, (i) the amplitude of the X-ray variability ( $\mathrm{rms}=$ $40 \%$, red noise), (ii) the detection of pulsations with a high pulse fraction $(P F \gtrsim 50 \%)$, (iii) the power-law component $(\Gamma=1.5)$, (iv) the coupling between periodic and aperiodic variability, and v) the small emitting area strongly suggest that X-rays from IGR J06074+2205 detected during the XMM-Newton observation result from accretion. The question that remains to be answered is how accretion could proceed when the main source of matter was exhausted.

Let us now examine the possibility of accretion from a stellar wind. Be stars, by definition, are dwarf, subgiant or giant objects (luminosity class III-V). Consequently, the stellar wind is weak. Assuming typical parameters $R_{X}=10^{6} \mathrm{~cm}, M_{\mathrm{X}}=1.4 M_{\odot}$, $M_{*}=15 M_{\odot}($ as expected for a B0.5V star Reig et al. 2010), $M_{\text {wind }}^{*} \sim 1 \times 10^{-8} \quad M_{\odot} \mathrm{yr}^{-1}$ (Prinja 1989; Vink et al. 2000), $v_{\text {wind }} \sim v_{\text {esc }} \approx 8.5 \times 10^{8} \mathrm{~cm} \mathrm{~s}^{-1}$, and $P_{\text {orb }}=100$ days, the X-ray luminosity from the stellar wind is well below $10^{33} \mathrm{erg} \mathrm{s}^{-1} . L_{\text {wind }}$ approaches the observed X-ray luminosity only if $P_{\text {orb }} \lesssim 10$ days or $v_{\text {wind }}$ is abnormally low $\left(400-500 \mathrm{~km} \mathrm{~s}^{-1}\right)$. BeXBs have orbital periods well above 20 days. From the $P_{\text {orb }}-P_{\text {spin }}$ relationship (Corbet 1986; Reig 2011), a system with $P_{\text {spin }}=373$ s should have $P_{\text {orb }} \gtrsim 100$ days. An exception is SAX J2103.5+4545, which is the $\mathrm{BeXB}$ with the shortest orbital period of 12.7 days but has a pulse period of $358 \mathrm{~s}$ (Baykal et al. 2002). Abnormally slow winds have been reported in one BeXB, 4U 2206+54 (Ribó et al. 2006). However, $4 \mathrm{U} 2206+54$ is a rather peculiar object (Blay et al. 2006). Reig \& Zezas (2014) argued that a weak and small disk might be present in the BeXB IGR J21343+4738 even though an absorption $\mathrm{H} \alpha$ profile was observed. A low-velocity outflow from this highly debilitated disk with $v_{\text {wind }} \sim 300-500 \mathrm{~km} \mathrm{~s}^{-1}$ could explain the X-ray luminosity of $\sim 10^{35} \mathrm{erg} \mathrm{s}^{-1}$, measured in that system. Accretion from an invisible (in the optical and IR bands) disk was also proposed by Ikhsanov (2001) to explain the quiescent emission of $1 \mathrm{~A} 0535+262$. We conclude that direct accretion from stellar wind cannot explain the origin of the X-ray emission in IGR J06074+2205, although a slow outflow from a weak optically undetectable equatorial disk cannot be ruled out. The fact that the $\mathrm{H} \alpha$ line showed clear evidence for emission less than two months after the XMM-Newton observation and that the $\mathrm{EW}(\mathrm{H} \alpha)$ was slightly smaller than that expected from a fully photospheric line possibly indicate the presence of a very weak residual disk.

The alternative to direct accretion is the formation of an accretion disk. The centrifugal barrier caused by the rotating magnetosphere is strongly suppressed if an accretion disk is present. Such a disk will form if the source luminosity is below the limiting luminosity given in Eq. (5). If this luminosity is reached before the propeller minimum luminosity the source will transit to stable accretion from an entirely recombined cold disk (Tsygankov et al. 2017a). For a typical X-ray pulsar with a magnetic field around $2 \times 10^{12} \mathrm{G}$, $L_{\text {disk }} \sim 2 \times 10^{34} \mathrm{erg} \mathrm{s}^{-1} \mathrm{erg} \mathrm{s}^{-1}$, while $L_{\text {min }}$ would be two orders of magnitude lower. Hence $L_{\min }<L_{\text {disk }}$, and the condition under which the source may accrete matter from the cold disk is met.

The long spin period of IGR J06074+2205 and the detection of X-ray pulsations put this source along with $1 \mathrm{~A} 0535+26$, $4 \mathrm{U} 1145-619$, and 1A 1118-615, in a category of systems with accretion powered quiescent emission.

\section{Conclusion}

We perform an X-ray timing and spectral analysis of the BeXB candidate IGR J06074+2205. We discovered pulsations with a pulse period of 373.2 s. Hence, our observations convert IGR J06074+2205 from a candidate BeXB to a confirmed accreting pulsar. We also found strong coupling between the periodic and aperiodic variability, which manifests itself as a broadening of the base of the pulse peak in the power spectrum. The $\mathrm{X}$-ray spectrum is well described by a thermal (blackbody) and a nonthermal (power law) components, affected by interstellar absorption. We did not find evidence for iron emission at $6.4 \mathrm{keV}$. The presence of the nonthermal component (interpreted as coming from bulk Comptonization), the strong red noise in the power spectrum, the pulsations, and the small emitting area of the thermal component (polars caps) are all considered as signatures of accretion and rule out cooling of the accretionheated neutron star crust as the sole mechanism that explains our XMM-Newton observation. Although accretion powers the quiescent emission in IGR J06074+2205, we cannot tell whether the source of accreted matter is a slow wind coming out from a weak decretion disk or from a recombined cold accretion disk. We should emphasize that the order of magnitude calculations presented in this work strongly depend on the magnetic fields strength. In the absence of a direct measurement, for example, through the detection of a cyclotron resonant scattering feature, these numbers should be taken with caution.

Acknowledgements. We thank N. Schartel for his fast response to our request to observe the source and for approving a TOO observation under Discretionary time. Skinakas Observatory is run by the University of Crete and the Foundation for Research and Technology-Hellas. A.Z. acknowledges funding from the European Union's Seventh Framework Programme (FP/2007-2013)/ERC Grant Agreement No. 617001.

\section{References}

Anders, E., \& Grevesse, N. 1989, Geochim. Cosmochim. Acta, 53, 197

Baykal, A., Stark, M. J., \& Swank, J. H. 2002, ApJ, 569, 903

Blay, P., Negueruela, I., Reig, P., et al. 2006, A\&A, 446, 1095

Brown, E. F., Bildsten, L., \& Rutledge, R. E. 1998, ApJ, 504, L95

Burderi, L., Robba, N. R., La Barbera, N., \& Guainazzi, M. 1997, ApJ, 481, 943

Campana, S., Gastaldello, F., Stella, L., et al. 2001, ApJ, 561, 924

Campana, S., Stella, L., Israel, G. L., et al. 2002, ApJ, 580, 389

Chenevez, J., Budtz-Jorgensen, C., Lund, N., et al. 2004, ATel, 223

Corbet, R. H. D. 1986, MNRAS, 220, 1047

Corbet, R. H. D. 1996, ApJ, 457, L31

den Herder, J. W., Brinkman, A. C., Kahn, S. M., et al. 2001, A\&A, 365, L7

Frank, J., King, A., \& Raine, D. J. 2002, Accretion Power in Astrophysics: 3rd edn. (Cambridge: Cambridge University Press)

Geppert, U., Küker, M., \& Page, D. 2006, A\&A, 457, 937

Halpern, J. P., \& Tyagi, S. 2005, ATel, 682

Hickox, R. C., Narayan, R., \& Kallman, T. R. 2004, ApJ, 614, 881

Ikhsanov, N. R. 2001, A\&A, 367, 549

Illarionov, A. F., \& Sunyaev, R. A. 1975, A\&A, 39, 185

Jansen, F., Lumb, D., Altieri, B., et al. 2001, A\&A, 365, L1

Jaschek, C., \& Jaschek, M. 1987, The Classification of Stars (Cambridge: Cambridge University Press)

La Palombara, N., \& Mereghetti, S. 2006, A\&A, 455, 283

La Palombara, N., \& Mereghetti, S. 2007, A\&A, 474, 137

La Palombara, N., Sidoli, L., Esposito, P., Tiengo, A., \& Mereghetti, S. 2009, A\&A, 505, 947

Larsson, S. 1996, A\&AS, 117, 197

Lazzati, D., \& Stella, L. 1997, ApJ, 476, 267

Leahy, D. A. 1987, A\&A, 180, 275

Leahy, D. A., Darbro, W., Elsner, R. F., et al. 1983, ApJ, 266, 160

Mason, K. O., Breeveld, A., Much, R., et al. 2001, A\&A, 365, L36

Mukherjee, U., \& Paul, B. 2005, A\&A, 431, 667

Negueruela, I., Reig, P., Finger, M. H., \& Roche, P. 2000, A\&A, 356, 1003 
Paul, B., \& Naik, S. 2011, Bull. Astron. Soc. India, 39, 429

Prinja, R. K. 1989, MNRAS, 241, 721

Reig, P. 2011, Ap\&SS, 332, 1

Reig, P., \& Milonaki, F. 2016, A\&A, 594, A45

Reig, P., \& Zezas, A. 2014, MNRAS, 442, 472

Reig, P., Zezas, A., \& Gkouvelis, L. 2010, A\&A, 522, A107

Reig, P., Doroshenko, V., \& Zezas, A. 2014, MNRAS, 445, 1314

Reig, P., Nersesian, A., Zezas, A., Gkouvelis, L., \& Coe, M. J. 2016, A\&A, 590, A122

Ribó, M., Negueruela, I., Blay, P., Torrejón, J. M., \& Reig, P. 2006, A\&A, 449, 687

Rutledge, R. E., Bildsten, L., Brown, E. F., et al. 2007, ApJ, 658, 514

Stella, L., White, N. E., \& Rosner, R. 1986, ApJ, 308, 669

Strüder, L., Briel, U., Dennerl, K., et al. 2001, A\&A, 365, L18

Syunyaev, R. A., \& Shakura, N. I. 1977, Sov. Astron. Lett., 3, 138

Tomsick, J. A., Chaty, S., Rodriguez, J., Walter, R., \& Kaaret, P. 2006, ATel, 959

Tsygankov, S. S., Lutovinov, A. A., Doroshenko, V., Mushtukov, A. A., \& Poutanen, J. 2016, A\&A, 593, A16
Tsygankov, S. S., Mushtukov, A. A., Suleimanov, V. F., et al. 2017a, A\&A, 608, A17

Tsygankov, S. S., Wijnands, R., Lutovinov, A. A., Degenaar, N., \& Poutanen, J. 2017b, MNRAS, 470, 126

Turner, M. J. L., Abbey, A., Arnaud, M., et al. 2001, A\&A, 365, L27

van der Klis, M. 1989, in NATO Advanced Science Institutes (ASI) Series C, (New York: Kluwer Academic/Plenum Publisher), eds. H. Ögelman, \& E. P. J. van den Heuvel, 262

Verner, D. A., Ferland, G. J., Korista, K. T., \& Yakovlev, D. G. 1996, ApJ, 465, 487

Vink, J. S., de Koter, A., \& Lamers, H. J. G. L. M. 2000, A\&A, 362, 295

Walter, R., Lutovinov, A. A., Bozzo, E., \& Tsygankov, S. S. 2015, A\&ARv, 23, 2

Waters, L. B. F. M., van den Heuvel, E. P. J., Taylor, A. R., Habets, G. M. H. J., \& Persi, P. 1988, A\&A, 198, 200

Wijnands, R., \& Degenaar, N. 2016, MNRAS, 463, L46

Wijnands, R., Degenaar, N., \& Page, D. 2013, MNRAS, 432, 2366

Yamamoto, T., Mihara, T., Sugizaki, M., et al. 2014, PASJ, 66, 59 